\title{
n-DIMENSIONAL FIRST INTEGRAL AND SIMILARITY SOLUTIONS FOR TWO-PHASE FLOW
}

\author{
S. W. WEEKS ${ }^{1}$, G. C. SANDER ${ }^{2}$ and J.-Y. PARLANGE ${ }^{3}$
}

(Received 12 May, 1998; revised 1 October, 1999)

\begin{abstract}
This paper considers similarity solutions of the multi-dimensional transport equation for the unsteady flow of two viscous incompressible fluids. We show that in plane, cylindrical and spherical geometries, the flow equation can be reduced to a weakly-coupled system of two first-order nonlinear ordinary differential equations. This occurs when the two phase diffusivity $D(\theta)$ satisfies $\left(D / D^{\prime}\right)^{\prime}=1 / \alpha$ and the fractional flow function $f(\theta)$ satisfies $d f / d \theta=\kappa D^{n / 2}$, where $n$ is a geometry index $(1,2$ or 3$), \alpha$ and $\kappa$ are constants and primes denote differentiation with respect to the water content $\theta$. Solutions are obtained for time dependent flux boundary conditions. Unlike single-phase flow, for two-phase flow with $n=2$ or 3 , a saturated zone around the injection point will only occur provided the two conditions $\int_{0}^{1} D /(1-f) d \theta<\infty$ and $f^{\prime}(1) \neq 0$ are satisfied. The latter condition is important due to the prevalence of functional forms of $f(\theta)$ in oil/water flow literature having the property $f^{\prime}(1)=0$.
\end{abstract}

\section{Introduction}

McWhorter and Sunada [9] presented a useful range of integral solutions to two-phase flow problems in both one and two dimensions based on the fractional flow function approach of McWhorter [8]. While these solutions are exact and apply for arbitrary capillary hydraulic properties, they do require iteration techniques to evaluate the saturation profiles. The one-dimensional solution of [9] is similar to that given by Sander et al. [19] which was based on the work of Parlange and Braddock [12] and Parlange [10]. The solution of [19] also applies for arbitrary capillary hydraulic

\footnotetext{
'School of Mathematical Sciences, Queensland University of Technology, GPO Box 2434, Brisbane QLD 400I, Australia; e-mail: s.weeks@fsc.qut.edu.au.

${ }^{2}$ Department of Civil and Building Engineering, Loughborough University, Loughborough, LE11 3TU, England; e-mail: G.Sander@lboro.ac.uk.

${ }^{3}$ Department of Biological and Environmental Engineering, Riley-Robb Hall, Cornell University, Ithaca, N.Y., USA; e-mail: jp58@cornell.edu.

(C) Australian Mathematical Society 2003, Serial-fee code 1446-1811/03
} 
properties and requires iteration to obtain the exact saturation profiles. However its advantage is that by incorporating the optimisation procedure of Parlange [11] an accurate first estimate of the saturation profile is obtained which renders iteration unnecessary.

There are now quite a few analytical closed-form exact solutions for two-phase flow. These are predominantly for one-dimensional flow problems and originate with Fokas and Yortsos [7] for $D=D_{0}(1-\nu \theta)^{-2},\left(D_{0}\right.$ and $\nu$ constants $), d f / d \theta=\kappa D$ and a constant flux boundary condition. Rogers et al. [16] then extended this to include the effect of gravity through a linear hydraulic conductivity function. Solutions were also obtained for two-phase absorption under Dirichlet boundary conditions by Sander $e t$ al. [18] using the same $D$ and $f$ as Fokas and Yortsos [7], and Sander et al. [17] with the same $D$ but with $f(\theta)=\left(f_{1}+f_{2} \theta+f_{3} \theta^{2}\right) /(1-\nu \theta)$, where $f_{1}, f_{2}$ and $f_{3}$ are constants.

In higher dimensions there seems to be only one exact explicit solution. This was found by Weeks et al. [24] in two dimensions, without gravity, for radially symmetric flow under a constant flux injection through a line source at $r=0$. Their solution can be written as

$$
\theta(r, t)= \begin{cases}\left(1-\alpha r^{2} /\left(4 D_{0} t\right)\right)^{1 / \alpha}, & r^{2}<4 D_{0} t / \alpha, \\ 0, & r^{2} \geq 4 D_{0} t / \alpha,\end{cases}
$$

for $D(\theta)=D_{0} \theta^{\alpha}, f(\theta)=\theta^{\alpha+1}$ and an injection flux $V=2 D_{0} /(\alpha+1)$. Their solution was found by extending the first integral approach of Parlange et al. [15] from single-phase to two-phase flow. In this paper we seek to use the same approach to obtain first integral style similarity solutions, $(\theta(\phi) ; \phi=r / \sqrt{t})$, for three-dimensional radially symmetric two-phase flow under a time dependent point source flux boundary condition. For completeness we present the first integral solutions for all symmetric geometries in one, two and three dimensions through a geometry index parameter $n$. As such, the previous solutions of Sander et al. [19], $n=1$, and Weeks et al. [24], $n=2$, will be obtainable directly from the theory presented here.

In $n$-dimensions, the nonlinear transport equation for radially symmetric two-phase flow is given by [5]

$$
\frac{\partial \theta}{\partial t}=\frac{1}{r^{n-1}} \frac{\partial}{\partial r}\left[r^{n-1} D(\theta) \frac{\partial \theta}{\partial r}-f(\theta) q_{t}\right],
$$

where $t$ is time, $r$ is the radial coordinate and $n=1,2$ and 3 for plane, cylindrical and spherical geometries. The boundary and initial conditions considered are

$$
\begin{array}{ll}
t=0, & r \geq 0, \theta=0, \\
t>0, & r=0, f q_{t}-r^{n-1} D(\theta) \partial \theta / \partial r=q_{w}(r=0, t)=q_{\omega 0}, \\
t>0, & r \rightarrow \infty, \theta \rightarrow 0 .
\end{array}
$$


In (1.2) and (1.3), $\theta(r, t)$ is the normalised volumetric water content with $0 \leq \theta \leq 1$ and is related to the actual water content $\Theta$ by $\theta=\left(\Theta-\Theta_{\mathrm{r}}\right) /\left(\Theta_{\text {sat }}-\Theta_{\mathrm{r}}\right)$, where $\Theta_{\text {sat }}$ and $\Theta_{\mathrm{r}}$ are the saturated and residual water contents respectively. The flux of water (or wetting fluid) is denoted by $q_{w}$, non-wetting fluid $q_{n w}$ and the total fluid flux $q_{t}=q_{w}+q_{n w}$. The volumetric flow rates $Q_{i}(i=w, n w, t)$ are related to their fluxes $q_{i}$ by $q_{i}=Q_{i} / G\left(\Theta_{\text {sat }}-\Theta_{\mathrm{r}}\right)$. Both $Q_{t}$ and the constant $G$ depend upon $n$. In 1,2 and 3 dimensions, $G$ equals $1,2 \pi b$ (where $b$ corresponds to the length of the line source), and $4 \pi$ respectively. For similarity, the water injection takes place along a plane, line or point source subject to [12]

$$
q_{w o}=V t^{n / 2-1},
$$

with $V$ a constant.

\section{First integral}

In this paper we will only be considering unidirectional displacement of a nonwetting phase by a wetting phase, therefore at $r=0, q_{n w}=0$ and $q_{t}=q_{w o}$. Defining the standard similarity variable

$$
\phi=r t^{-1 / 2},
$$

then (1.2) reduces to

$$
-\frac{\phi^{n}}{2} \frac{d \theta}{d \phi}=\frac{d}{d \phi}\left[\phi^{n-1} D \frac{d \theta}{d \phi}-f Q\right],
$$

while (1.3a) and (1.3c) coalesce to $\theta=0, \phi \rightarrow \infty$. The handling of boundary condition (1.3b) depends very much on $n$. Integrating (2.2) gives

$$
-\frac{1}{2} \int_{0}^{\infty} \phi^{n} \frac{d \theta}{d \phi} d \theta=\left[f V-D \frac{d \theta}{d \phi}\right]_{\theta=\theta_{s}}=V,
$$

where the last equality comes from combining (1.3b) and (1.4) and remembering $q_{t}=q_{w o}$. In the case of $n=1$, at $\phi=0, \theta=\theta_{s}$, where $\theta_{s}$ is constant with time and related to $V$ through (2.3) (since in this case $\theta(\phi)$ is invertible) by [19]

$$
\int_{0}^{\theta_{s}} \phi d \theta=2 V
$$

Here $V$ is related to the well-known sorptivity $S$ by $S=2 V$ and therefore (2.4) also imposes an upper limit to $V$ defined by $\theta_{s}=1$.

When we move to two- and three-dimensional flow regimes $(n=2,3)$ a saturated zone will usually develop around the source [9]. In this zone $\theta=1, d \theta / d \phi=0$ and 
$\theta(\phi)$ is not uniquely invertible. However if we define the edge of this saturated zone by $\phi=\phi_{1}$, then we can still invert the solution provided $\phi>\phi_{1}$ and then (2.3) and (2.4) are replaced by

$$
\left[f V-\phi^{n-1} D \frac{d \theta}{d \phi}\right]_{\phi=\phi_{1}}=V, \quad n=2,3
$$

where $\phi_{1}$ is obtained from

$$
-\int_{\phi_{1}}^{\infty} \phi^{n} \frac{d \theta}{d \phi} d \phi=\int_{0}^{1} \phi^{n} d \theta=2 V, \quad n=2,3 .
$$

To obtain a first integral solution we follow the method as outlined in Parlange et al. [13-15] and define the transformations

$$
u=\phi D^{-1 / 2}
$$

and

$$
d y=d D / D
$$

where $y$ is the independent variable. Equation (2.2) then becomes

$$
\begin{aligned}
\frac{u}{2}\left(\frac{u}{2}+u^{\prime}\right)^{2} & -\left(\frac{u^{\prime}}{2}+u^{\prime \prime}\right)-\frac{V D^{-n / 2}}{u^{n-1}}\left(\frac{u}{2}+u^{\prime}\right)^{2} \frac{d f}{d \theta} \\
& +\left(\frac{u}{2}+u^{\prime}\right)\left(\frac{n}{2}+(n-1) \frac{u^{\prime}}{u}\right)+\left(\frac{u}{2}+u^{\prime}\right) h(\theta)=0,
\end{aligned}
$$

where

$$
h(\theta)=\frac{d}{d \theta}\left[\frac{D}{d D / d \theta}\right]
$$

If (2.7) is to lead to a first integral, then it is necessary that both the functions $h(\theta)$ and $V D^{-n / 2} d f / d \theta$ be independent of $\theta$. This is easily achieved by setting $h(\theta)=1 / \alpha$ and $V D^{-n / 2} d f / d \theta=\beta$, where $\alpha$ and $\beta$ are constants. It is hardly surprising that the condition $h(\theta)=1 / \alpha$ emerges from our analysis. It is well-known that this is the necessary condition for Lie group invariance of the nonlinear diffusion equation and Richards' equation $[2,21,22]$ which is a property that known exact solutions possess. Integrating (2.8) shows that $D(\theta)$ must be of the form

$$
D(\theta)=D_{0}(\theta+\mu)^{\alpha}
$$

or

$$
D(\theta)=A e^{p \theta},
$$


where $\mu, A$ and $p$ are positive constants, and (2.9b) is the limiting case of $\alpha \rightarrow \infty$. If we now set $V D^{-n / 2} d f / d \theta=\beta$ and integrate using $f(0)=0$ and $f(1)=1$ then $f(\theta)$ is given by

$$
f(\theta)=\frac{\int_{0}^{\theta} D^{n / 2} d \sigma}{\int_{0}^{1} D^{n / 2} d \theta},
$$

with

$$
\beta=\frac{V}{\int_{0}^{1} D^{n / 2} d \theta},
$$

and $D(\theta)$ given by either (2.9a) or (2.9b) only.

The form of (2.7) suggests we take a new function $\omega([13])$

$$
\omega=u / 2+u^{\prime}
$$

which combined with (2.9)-(2.11) allows (2.7) to be rewritten as the first-order differential equation

$$
\left[u+\frac{2}{u}\left(n-1-\beta u^{2-n}\right)\right] \omega^{2}+\left(1+\frac{2}{\alpha}\right) \omega-(2 \omega-u) \frac{d \omega}{d u}=0 .
$$

Therefore whenever $D$ and $f$ obey (2.9) and (2.10) a first integral exists. Equations (2.12) and (2.13) form a weakly-coupled system of first-order differential equations for the function $u(y)$. First consider the case of the exponential diffusivity as given by (2.96). When $D \neq 0$ at $\theta=0$, as in an exponential $D, \phi \rightarrow 0$ asymptotically as $\theta \rightarrow 0$. From (2.6a) $\phi \rightarrow \infty$ implies $u \rightarrow \infty$ and (2.13) can be approximated by $u \omega^{2}-2 \omega d \omega / d u \approx 0$ which has the dominant solution $\omega \approx \gamma \exp \left(u^{2} / 4\right)$ with $\gamma \neq 0$. Clearly $\gamma$ must depend not only on $p$ in (2.9b) but also the surface flux $V$. Unfortunately there is no direct means of determining this dependence other than through iteration. However when $D(0)=0$, iteration is unnecessary and $\gamma$ is known directly, given by $\gamma=0$ [12]. In this case it can be shown that as $u \rightarrow 0, \omega \rightarrow 0$ also.

In this paper we are only interested in solutions which do not require iteration and from now on we only consider $D(\theta)$ as in (2.9a) with $\mu=0$ so that $D(0)=0$. Thus from (2.9a), (2.10) and (2.11)

$$
\begin{aligned}
D(\theta) & =D_{0} \theta^{\alpha}, \\
f(\theta) & =\theta^{n \alpha / 2+1}, \\
\beta & =V(n \alpha / 2+1) D_{0}^{-n / 2} .
\end{aligned}
$$

Since $D(0)=0, \phi$ at $\theta=0$ must be finite, $u \rightarrow \infty$ and $\omega^{-1}$ can be expanded as a series in powers of $u$ in the form

$$
\omega^{-1}=-\alpha u / 2+A_{n}+B_{n} u^{-1}+C_{n} u^{-2}+E_{n} u^{-3}+O\left(u^{-4}\right) .
$$


where $A_{n}, B_{n}, C_{n}$ and $E_{n}$ are constants which depend on the dimension $n$. The importance of $(2.17)$ is that it provides the necessary boundary condition for the integration of (2.13) and therefore (2.12). If we substitute into (2.13) we obtain

$$
\begin{aligned}
\left(\frac{2}{\alpha}+1\right) A_{n} & -2 \beta u^{1-n}+2\left(n+\left(\frac{1}{\alpha}+1\right) B_{n}\right) u^{-1}+\left(\left(\frac{2}{\alpha}+3\right) C_{n}+\frac{4}{\alpha} A_{n}\right) u^{-2} \\
+ & {\left[\left(\frac{2}{\alpha}+4\right) E_{n}+\frac{8}{\alpha}\left(B_{n}+\frac{A_{n}^{2}}{\alpha}\right)\right] u^{-3}+O\left(u^{-4}\right)=0 }
\end{aligned}
$$

For (2.18) to hold true, all coefficients of $u$ must be zero. Thus, by setting $n$ and grouping like terms, we find that the constants $A_{n}, B_{n}, C_{n}$ and $E_{n}$ take on the following values: for $n=1$,

$$
\begin{array}{ll}
A_{1}=\frac{2 \alpha \beta}{\alpha+2} . & C_{1}=\frac{-8 \alpha \beta}{(\alpha+2)(3 \alpha+2)}, \\
B_{1}=\frac{-\alpha}{\alpha+1}, & E_{1}=\frac{4 \alpha\left[(\alpha+2)^{2}-4 \beta^{2}(\alpha+1)\right]}{(\alpha+1)(2 \alpha+1)(\alpha+2)^{2}},
\end{array}
$$

for $n=2$,

$$
A_{2}=0, \quad B_{2}=\frac{\alpha(\beta-2)}{\alpha+1}, \quad C_{2}=0, \quad E_{2}=\frac{-4 \alpha(\beta-2)}{(\alpha+1)(2 \alpha+1)}
$$

and for $n=3$,

$$
A_{3}=0, \quad B_{3}=\frac{-3 \alpha}{\alpha+1}, \quad C_{3}=\frac{2 \alpha \beta}{3 \alpha+2}, \quad E_{3}=\frac{12 \alpha}{(\alpha+1)(2 \alpha+1)} .
$$

For $n=2$, the coefficients of all of the even powers of $u\left(u^{0}, u^{-2}\right.$, etc. $)$ are zero and for $\beta=2, \omega=-2 / \alpha u$ is the solution of (2.13). This leads to the fully analytic solution for (1.1) in cylindrical geometry given by $\theta(r, t)=\left(1-\alpha r^{2} / 4 D_{0} t\right)^{1 / \alpha}$ for $r^{2}<4 D_{0} t / \alpha$ and $\theta(r, t)=0$ for $r^{2} \geq 4 D_{0} t / \alpha$ [24]. No similar phenomena are found in either the plane or spherical cases.

The definitions of $D(\theta)$ and $y$ from (2.14) and (2.6b) allow (2.12) to be written as

$$
\frac{d u}{d \ln \theta}=\alpha\left(\omega-\frac{u}{2}\right)
$$

which can be integrated to first order for $u$ large $(\omega \rightarrow 0)$ to give $u \approx c \theta^{-\alpha / 2}$ (c being a constant) which combined with the definition of $u$ gives $\phi=u D^{1 / 2} \approx c \theta^{-\alpha / 2} D_{0}^{1 / 2} \theta^{\alpha / 2}$ being finite at $\theta=0$. Consequently a well-defined wetting front is moving into the soil, consistent with $D(0)=0$. Equation $(2.17)$ provides the starting condition at infinity (the position of the wetting front) so that (2.13) can be integrated backwards in the direction $u \rightarrow 0$. The initial value of $u$ has to be chosen large enough so that 
the higher order terms in (2.17) become negligible. The boundary condition given by either (2.3) or (2.5) depending on $n$, determines how far (2.13) is integrated backwards. Rewriting (2.5) as

$$
V=-\phi^{n-1} D_{w}(\theta) \frac{d \theta}{d \phi}, \quad \phi=\phi_{1}
$$

then $D_{w}(\theta)=D(\theta) /(1-f)$ is the usual single-phase (water) diffusivity.

Substituting (2.6a) for $u$ in (2.20) and expanding the derivative term will give the following expression for the flux in terms of $u$ and $\omega$ at the injection point $\phi=\phi_{1}$,

$$
-\left.\phi^{n-1} D_{w}(\theta) \frac{d \theta}{d \phi}\right|_{\phi=\phi_{1}}=\left[\frac{-u^{n-1} D_{0}^{n / 2} \theta^{n \alpha / 2+1}}{\alpha \omega(1-f)}\right]_{\phi=\phi_{1}},
$$

or combining with (2.21)

$$
V=\left[\frac{-u^{n-1} D_{0}^{n / 2} \theta^{n \alpha / 2+1}}{\alpha \omega(1-f)}\right]_{\phi=\phi_{1}} .
$$

For $n=1, \theta=\theta_{s}$ at $\phi=0$ and with $f(\theta)$ from (2.15)

$$
V=\frac{D_{0}^{1 / 2}}{\alpha \omega(0)\left(1-\theta_{s}^{-(\alpha / 2+1)}\right)} .
$$

As $V=S / 2,(2.24)$ provides a relationship between the sorptivity $S$ and $\theta_{s}$ and is equivalent to (2.14) of Sander et al. [19].

For $n=2$ or 3 , however, $\theta=1$ at $\phi=\phi_{1}$ which means that the denominator of the right-hand side of $(2.23)$ is zero due to $f(1)=1$. Therefore, if we are to obtain a finite flux $V$ from (2.23), then $u$ must also be zero. The definitions of $\phi$ and $u$ from (2.1) and (2.6a) show that $u=0$ corresponds to $r=0$. This means that no saturation zone develops around the source for the two- and three-dimensional problems. This occurs because of the functional form of $D$ and $f$ in (2.14) and (2.15) resulting in the sorptivity $S$, approximated by [11]

$$
\left[2 \int_{0}^{1} \frac{D}{1-f} d \theta\right]^{1 / 2}
$$

being infinite. Consequently any flux of water injected at the source $(r=0)$ can be absorbed by the soil and no saturation zone forms. This is by no means the only restriction on the formation of a saturated zone. A saturated zone will also not occur for $n=2$ and 3 if $f(\theta)$ has the property $f^{\prime}(1)=0$ even if $S$ is finite. A proof and further discussion of saturated zone formation is given in a later section. 


\section{Asymptotic expansions for $\theta(\phi \rightarrow 0)$}

We can obtain expansions for $\theta(\phi)$ as $\phi \rightarrow 0$ or when $\theta$ is near $\theta_{s}$ for $n=1$, and $\theta$ near 1 (saturation) for $n=2$ and $n=3$.

Case $n=1$. The differential equation for $n=1$ is

$$
(2 \omega-u) \frac{d \omega}{d u}=\omega\left[(u-2 \beta) \omega+\frac{2}{\alpha}+1\right]
$$

and we look for an expansion for $\omega(u \rightarrow 0)$ of the form

$$
\omega=\omega_{0}\left(1+a_{1} u+c_{1} u^{2}+O\left(u^{3}\right)\right)
$$

where $\omega_{0}=\omega(u=0)$. Substituting (3.2a) into (3.1) and by matching terms of the order $u^{0}$ and $u$ we find

$$
a_{1}=-\beta+(1 / 2+1 / \alpha) \omega_{0}^{-1}
$$

and

$$
c_{1}=a_{1}(2+2 / \alpha)\left(4 \omega_{0}\right)^{-1}+1 / 4-a_{1} \beta-a_{1}^{2} / 2 .
$$

An estimate of $\omega_{0}$ for arbitrary $\alpha$ is not possible, however by following Parlange et al. [13] we can find estimates to $\omega_{0}$ for $\alpha$ large. The exponent $\alpha$ is usually large for soils, for example $\alpha \geq 4$, and we note from (2.17) that for large $\alpha, u \omega$ behaves like $\alpha^{-1}$. Substituting for $u \omega$ into the right-hand side of (3.1) and then neglecting $O\left(\alpha^{-1}\right)$ terms, (3.1) simplifies to

$$
\frac{d \omega}{d u}=\frac{\omega-2 \beta \omega^{2}}{2 \omega-u}
$$

Integrating (3.1) along with the boundary condition $u \omega \rightarrow-2 / \alpha$ as $u \rightarrow \infty$, an estimate for $\omega_{0}$ is obtained by solving the nonlinear equation

$$
\frac{1-2 \beta \omega_{0}}{2 \beta^{2}} \ln \left(1-2 \beta \omega_{0}\right)-\frac{2}{\alpha}+\left(\frac{4 \beta}{\alpha}+\frac{1}{\beta}\right) \omega_{0}=0 .
$$

Finally combining (2.6a), (2.14), (2.20) and (3.2a) and keeping only the dominant first two terms shows that as $\phi \rightarrow 0, \theta(\phi)$ behaves like $\theta=\theta_{s}+\left(\alpha \omega_{0} D_{0}^{1 / 2} \theta_{s}^{\alpha / 2}\right)^{-1} \phi$, with $\omega_{0}$ estimated from (3.3).

Case $n=2$. In two dimensions, the first-order approximation for $\omega(u)$ is given by $\omega=\omega_{0} u^{1-\beta}[24]$ and we look for the second-order term in the form

$$
\omega=\omega_{0} u^{1-\beta}+c_{2} u^{d}
$$




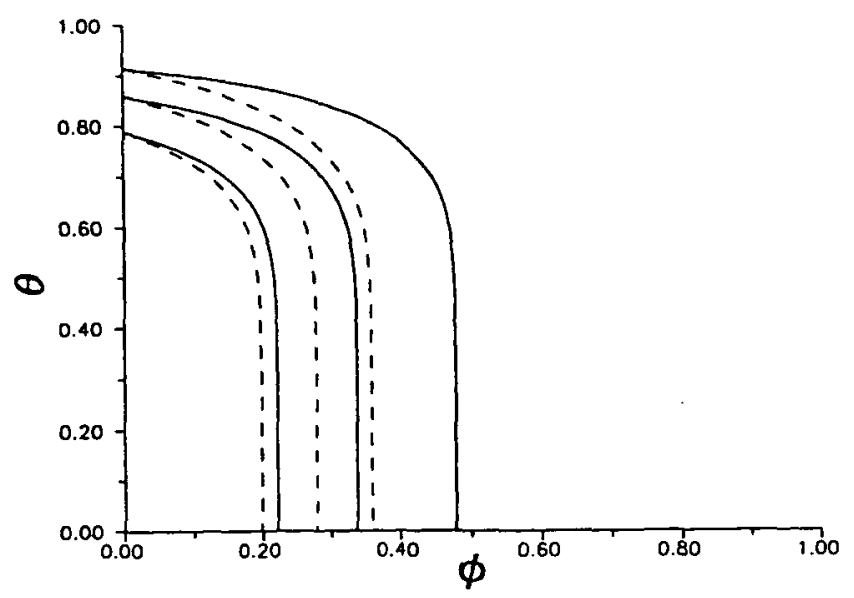

FIGURE 1. Profiles of $\theta(\phi)$ when $n=1$ for one- and two-phase flow with $\alpha=8$ and $\theta_{s}$ equivalent to $\beta=0.5,1$ and 2 .

where the constants $c_{2}$ and $d$ can be found by substituting (3.4) into (2.13) and grouping like terms. For consistency we find $d=1$ and the two-term expansion for $\omega(u)$ is $\omega=\omega_{0} u^{1-\beta}+(1 / 2)(2-\beta+2 / \alpha) u / \beta$. For $n=2$, it is also possible to get a first-order estimate of $\omega_{0}$ for the cases where $\alpha$ is large. As for $n=1$, large $\alpha$ means that we can take $u \omega \approx-2 / \alpha$, then substituting into (2.22) and neglecting terms of $O\left(\alpha^{-1}\right)$ we have

$$
\frac{d \omega}{d u}=\frac{2(1-\beta) \omega / u+1}{2-u / \omega},
$$

which can be integrated with the boundary condition $u \omega \rightarrow 2 / \alpha$ as $u \rightarrow \infty$, resulting in the first-order approximation

$$
\omega_{0}=-\frac{1}{\beta}\left(\frac{2 \beta}{\alpha}\right)^{\beta / 2} .
$$

The next term in the series can also be found by using the solution of (3.5) to estimate the $O\left(\alpha^{-1}\right)$ terms initially neglected. For $1<\beta<\alpha^{2}$ the two-term expansion for $\omega_{0}$ is

$$
\omega_{0}=-\frac{1}{\beta(1-1 / \alpha)}\left(\frac{2 \beta(1-1 / \alpha)}{\alpha}\right)^{\beta(1-1 / \alpha) / 2} .
$$

It should also be noted that for $\beta=1$, the differential equation for $\omega(u)$ reduces to (13) of [13]. Hence, a three-term expansion for $\omega_{0}$ in this case ( $\beta=1, \alpha$ large) is given by (23) of [13], that is,

$$
\omega_{0}^{2}=2 \alpha^{-1}+3 \alpha^{-2}+\left(6 \alpha^{3}\right)^{-1} .
$$




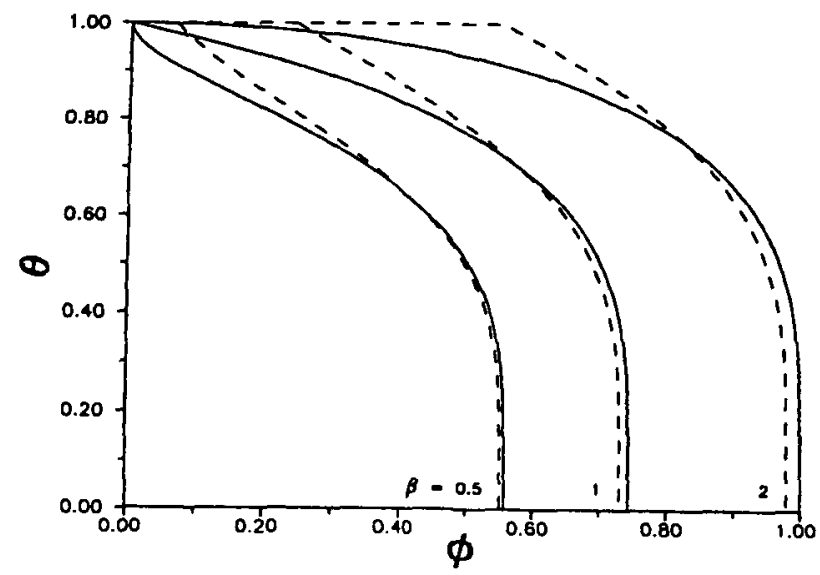

FIGURE 2. Profiles of $\theta(\phi)$ for $n=2, \alpha=4$ and $\beta$ as marked.

The expansions for $\theta(\phi \rightarrow 0)$ as $\phi \rightarrow 0$ is given by combining (2.6a), (2.14), (2.20) and (3.4) as [24]

$$
\theta \approx 1+\frac{D_{0}^{-\beta / 2}}{\alpha \omega_{0} \beta} \phi^{\beta}
$$

By differentiating (3.6) it is possible to see that in two dimensions the value of $\beta$ determines the behaviour of $d \theta / d \phi$ as $\phi \rightarrow 0$. There are three distinct types of behaviour of $d \theta / d \phi$, (i) $d \theta / d \phi \rightarrow-\infty$ for $0<\beta<1$, (ii) $d \theta / d \phi \rightarrow\left(D_{0}^{1 / 2} \alpha \omega_{0}\right)^{-1}$ for $\beta=1$ and (iii) $d \theta / d \phi \rightarrow 0$ for $\beta>1$. The case $0<\beta<1$ is particularly interesting as it implies a rapid decline in the water content away from the injection point and that overall an S-shaped water profile will result. This type of behaviour has often been noticed in two-phase flow experiments (see Bond and Collis-George [3], for example). The definition of $\beta$ shows that $\mathrm{S}$-shaped profiles will predominantly occur under small injection rates $V$, which is also in agreement with the findings of McWhorter and Sunada [9].

Case $n=3$. In three dimensions, the differential equation for $d \omega / d u$ is

$$
\frac{d \omega}{d u}=\frac{\omega}{2 \omega-u}\left[\left(u+\frac{2}{u}\left(2-\frac{\beta}{u}\right)\right) \omega+\frac{2}{\alpha}+1\right] .
$$

For $u$ small the dominant term in (3.7) is given through $(2-\beta / u) \omega / u$. Neglecting other terms and integrating gives a first-order approximation for $\omega$ as

$$
\omega=\omega_{0} u^{2} e^{\beta / u}
$$




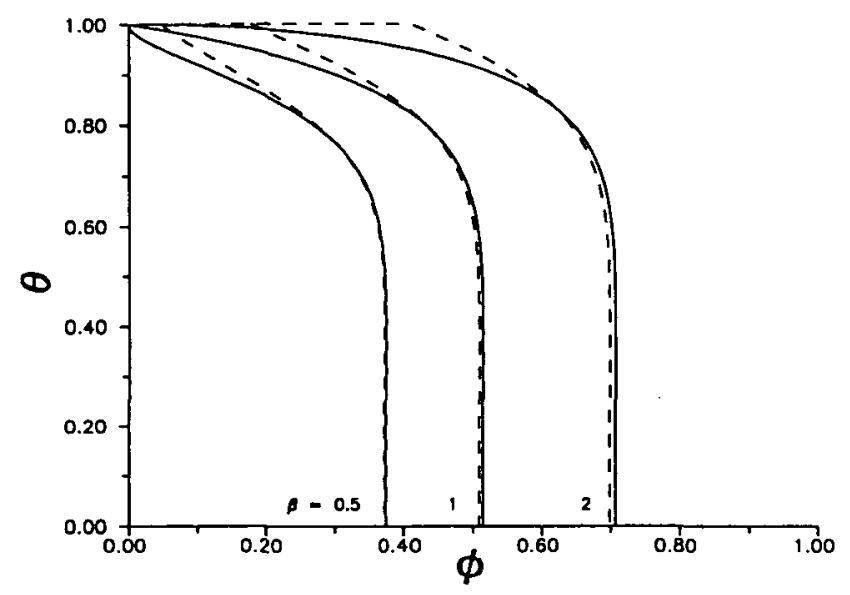

FIGURE 3. Profiles of $\theta(\phi)$ for $n=2, \alpha=8$ and $\beta$ as marked.

Then from (2.6a), (2.14), (2.20) and (3.8) the first-order approximation to $\theta$ for $\phi \rightarrow 0$ is

$$
\theta=1+\left(\alpha \omega_{0} \beta\right)^{-1} e^{-\beta /\left(D_{0}^{1 / 2} \phi\right)} .
$$

Differentiating (3.9) shows that $d \theta / d \phi$ at $\phi=0$ for $n=3$ is always zero. As yet estimates to $\omega_{0}$ for $n=3$ have not been found.

\section{Discussion}

In general, (2.12) and (2.13) cannot be integrated analytically. Using (2.17) as the starting condition (2.13) is integrated backwards to $u=0$ using the fourth-order Runge-Kutta method to obtain $\omega(u)$. Having $\omega(u),(2.12)$ can be integrated using Simpson's rule for $u(y)$ using the boundary condition $u=0, y=\alpha \ln \left(\theta_{s}\right)$ for $n=1$, or $u=0, y=0$ for $n=2$ and 3. Equation (2.6) then finally gives $\phi(\theta)$ or $\theta(\phi)$ parametrically. When $\beta>1$ for $n=2$ and 3, (3.4) and (3.8) show that $\omega \rightarrow-\infty$ as $u \rightarrow 0$, then for these cases the integration is taken back only as far as $u=u^{*}$, $u^{*}>0$. When $\omega(u)$ is known numerically, (3.4) when $n=2$ or (3.8) when $n=3$ can be rearranged to estimate $\omega_{0}$ numerically. As $u \rightarrow 0$ these expansions become more accurate, thus $u^{*}$ is defined by the value of $u$ giving $\omega_{0}$ accurate to four significant figures. Equation (2.12) is still integrated as before but with the expansions used for $\omega(u)$ when $0 \leq u \leq u^{*}$ and the numerical solution for $u>u^{*}$. All figures presented in this section are accurate to four significant figures.

Figures 1 to 6 show the $\theta(\phi)$ profiles as a function of $\alpha$ and $V$ for all three dimensions. We have also included in these figures the one-phase moisture profiles 


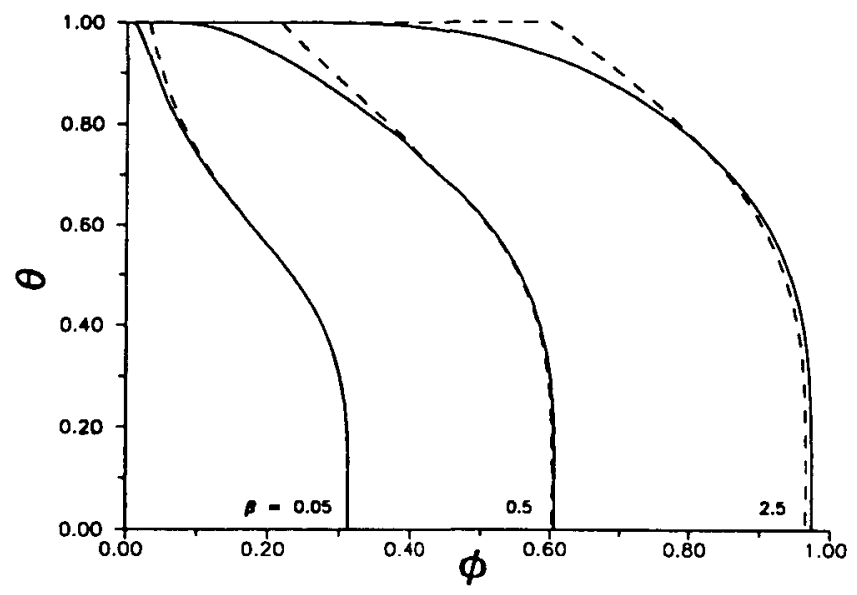

FIGURE 4. Profiles of $\theta(\phi)$ for $n=3, \alpha=4$ and $\beta$ as marked.

(dashed lines) for the purposes of comparison. The single-phase $n$-dimensional flow equations are given in Parlange and Braddock [12] and can be obtained directly from (2.13) by setting $\beta=0$. However in both two- and three-dimensional one-phase flow conditions a saturated zone will develop around $r=0$ in which case (2.13) (with $\beta=0$ ) is integrated only as far back as $u=u_{1}, \omega=\omega_{1}$, where $u_{1}, \omega_{1}$ satisfy [12]

$$
\omega_{1}^{-1} u^{n-1}=-\alpha V D_{0}^{-n / 2} .
$$

Note that (4.1) is obtainable directly from (2.23) by simply taking $f=0$. If $n=1$, then (2.24) is replaced by

$$
\theta_{s}^{1+\alpha / 2}=-\alpha V \omega(0) D_{0}^{-1 / 2}
$$

In Figure $1(n=1)$ both the one- and two-phase profiles (solid line) are plotted for the same surface water content $\theta_{s}$, hence the fluxes $V$ will be different and given by (4.2) for single-phase and (2.24) for two-phase flow. In Figures 2 to 6 both the single and two-phase flow profiles (solid lines) have the same injection flux $V$ determined from (2.16) as $V=\beta D_{0}^{n / 2}(1+n \alpha / 2)^{-1}$ once $\beta, \alpha$ and $n$ are given. Without loss of generality $D_{0}$ is taken as 1 in all the figures.

Figures 2 to 6 show that the effect of increasing $\alpha$ or increasing geometry dimension through $n$ is to reduce the influence of the non-wetting phase on the saturation profile $\theta(\phi)$. Secondly the effect of the non-wetting phase is predominantly to alter the shape and position of the saturation profile around $\theta=1$ and it has very little influence over the position or shape of the tail of the wetting front itself. The reason for this is clearly seen when we look at the functional form of $f(\theta)$, that is, $f(\theta)=\theta^{1+n \alpha / 2}$. As either 


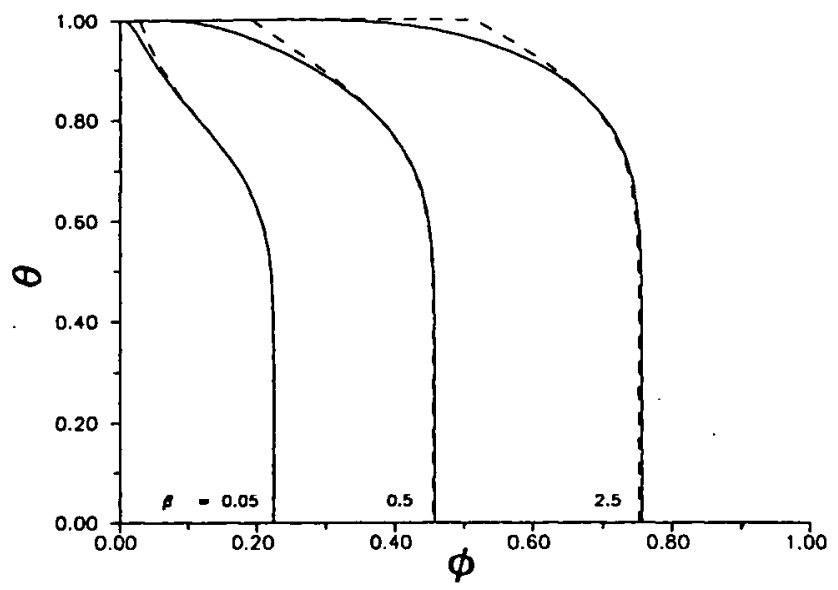

FIGURE 5. Profiles of $\theta(\phi)$ for $n=3, \alpha=8$ and $\beta$ as marked.

$\alpha$ or $n$ increases and in particular for $\alpha$ large, $f(\theta)$ will only be significantly different from 0 for $\theta$ near 1 . Thus $f(\theta)$ starts to behave like a delta function and therefore the non-wetting phase has little or no influence on the saturation profile.

The other feature we notice in Figures 2 to 6 is the $S$-shaped profiles which occur when $n=2$ or 3. For $n=2$ these profiles will occur for $\beta<1$ (Equation (3.6)) or for a flux $V \leq(1+\alpha)^{-1}$. These profiles therefore only arise for small injection fluxes, and the larger the diffusivity exponent $\alpha$, the smaller the flux $V$ will need to be. It is also clear that $S$-shaped profiles will develop when $n=3$ for a small injection flux. However with $n=3$, while the value of $\beta$ necessary for $S$-shaped profiles is much smaller than its value when $n=2$, there is no limiting value of $\beta$ below which these profiles will always occur. This can be seen in Figures 4 and 5 for $\beta=0.05$, where for $\alpha=4$ the profile is markedly S-shaped but at $\alpha=8$ it is barely so and in fact disappears once $\beta$ increases to 0.1 .

The S-shaped profiles also appear for single-phase flow in two and three dimensions and are therefore not due to the presence of the second fluid. If anything, the effect of the second fluid has been to diminish the magnitude of the positive curvature in $\theta(\phi)$ for small $\phi$ when $n=2$ or 3 . We see this most strikingly in Figure 6 for $n=3, \alpha=2$ and $\beta=0.5$, where the single-phase profile is S-shaped and the two-phase profile is not.

Saturation zones. We saw from (2.23) that our two- and three-dimensional first integral solutions do not develop a saturated zone around the injection point. This is due in part to the diffusivity required for a first integral to exist, that is, (2.9) is not zero at $\theta=1$. While the traditional single-phase diffusivity $D_{w}$ is a monotonically increasing function of $\theta$, the two-phase diffusivity $D(\theta)$ is zero at both $\theta=0$ and 


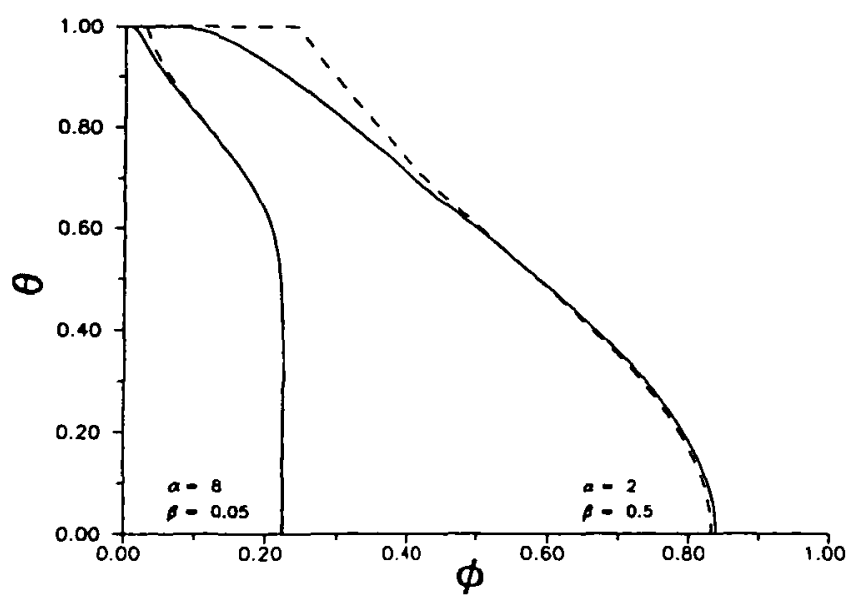

Figure 6. Profiles of $\theta(\phi)$ for $n=3$, with $\alpha$ and $\beta$ as marked.

$\theta=1$. With $D$ and $D_{w}$ related by $D_{w}=D /(1-f)$, then from (2.14) and (2.15) not only does $D_{w}(1) \rightarrow \infty$ but also

$$
\int_{0}^{1} D_{w} d \theta \rightarrow \infty
$$

For $D_{w}$ increasing rapidly with $\theta$ we know from [11] that the integral in (4.3) is approximately $S^{2} / 2$. Consequently if the soil's sorptivity is infinite it will not be possible for a saturated zone to develop. It is quite straightforward to derive this condition for arbitrary $D(\theta)$ with $D(1)=0$ rather than just the particular form of $D(\theta)$ necessary for a first integral. Integrating (2.2) we have

$$
\psi(\theta)=\int_{0}^{\theta} \frac{D d \sigma}{V(1-f)-(1 / 2) \int_{\sigma}^{1} \phi^{n} d \rho},
$$

where

$$
\psi(\theta)= \begin{cases}\phi, & \text { for } n=1, \\ \ln \left(\phi_{f} / \phi\right), & \text { for } n=2, \\ \phi^{-1}-\phi_{f}^{-1}, & \text { for } n=3\end{cases}
$$

with $\phi_{f}$ being the position of the wetting front. Without loss of generality we assume $D(0)=0$ so that $\phi_{f}$ is finite. Since $\phi(\theta)$ is a monotonically decreasing function of $\theta$ we know that the integrand in (4.4) is always positive, hence

$$
V(1-f) \geq \frac{1}{2} \int_{\theta}^{1} \phi^{n} d \sigma
$$


and therefore at $\theta=1(4.4)$ has the lower bound

$$
\int_{0}^{1} \frac{D d \theta}{V(1-f)-(1 / 2) \int_{\theta}^{1} \phi^{n} d \rho} \geq \frac{1}{V} \int_{0}^{1} D_{w} d \theta .
$$

If the single-phase diffusivity $D_{w}$ satisfies (4.3), then from (4.5) and (4.6), $\psi \rightarrow \infty$ and the position of the saturated zone $\phi_{1}\left(\theta=1, \phi=\phi_{1}\right)$ must be at zero.

In single-phase flow, having the integral in (4.7) being finite is sufficient for a saturated zone to develop, however for two-phase flow a further condition is required. We see this by first Taylor expanding the integrand of (4.4) around $\theta=1$ as

$$
\begin{aligned}
& \frac{D}{V(1-f)-(1 / 2) \int_{\theta}^{1} \phi^{n} d \rho} \\
& \quad \approx \frac{D^{\prime}(1)+(\theta-1) D^{\prime \prime}(1) / 2}{V\left[\phi_{1}^{2} /(2 V)-f^{\prime}(1)\right]+(\theta-1)\left[\left.(n / 2) \phi_{1}^{n-1}(d \phi / d \theta)\right|_{\theta=1}-f^{\prime \prime}(1)\right] / 2} .
\end{aligned}
$$

Since (4.8) must be positive and $D^{\prime}(1) \leq 0$, then $\phi_{1}^{2} /(2 V) \leq f^{\prime}(1)$, so that if $f^{\prime}(1)=0, \phi_{1}$ must also be zero and again no saturated zone forms. It is worthwhile noting that it is very common in the oil/water and petroleum reservoir modelling literature to use functional forms of $f(\theta)$ which have the property $f^{\prime}(1)=0[1,4,6$, $20,23]$.

In conclusion we have extended the $n$-dimensional single-phase first integral similarity solutions of Parlange and Braddock [12] to two-phase flow when $D(\theta)=D_{0} \theta^{\alpha}$ and $f(\theta)=\theta^{1+n \alpha / 2}$. We have also shown that $S$-shaped saturation profiles develop at low injection fluxes $V$ in both two and three dimensions and that for a saturated zone to develop around the injection point $r=0$, the hydraulic functions $D(\theta)$ and $f(\theta)$ must satisfy the two conditions of $\int_{0}^{1} D /(1-f) d \theta<\infty$ and $f^{\prime}(1) \neq 0$.

\section{References}

[1] M. B. Allen, III, "Numerical modelling of multiphase flow in porous media", Adv. Water Resour. 8 (1980) 163-186.

[2] G. W. Bluman and S. Kumei, "On the remarkable nonlinear diffusion equation $(\partial / \partial x)(u(u+$ $\left.b)^{-2}(\partial u / \partial x)\right)-(\partial u / \partial t)=0 "$, J. Math. Phys. 21 (1980) 1019-1023.

[3] W. J. Bond and N. Collis-George, "Ponded infiltration into simple soil systems: 1. The saturation and transition zones in the moisture content profile", Soil Sci. Soc. Amer. J. 131 (1981) 202-209.

[4] M. A. Celia and P. Binning, "A mass conservative numerical solution for two-phase flow in porous media with application to unsaturated flow", Water Resour. Res. 28 (1992) 2819-282.

[5] Z. X. Chen, "Some invariant solutions to two-phase displacement problems including capillary effect", SPEJ May (1988) 691-700.

[6] C. R. Faust, J. H. Guswa and J. W. Mercer, "Simulation of three-dimensional flow of immiscible fluids within and below the unsaturated zone", Water Resour. Res. 12 (1989) 2449-2464. 
[7] A. S. Fokas and Y. C. Yortsos, "On the exactly solvable equation $S_{t}=\left[(\beta S+\gamma)^{-2} S_{x}\right]_{x}+\alpha(\beta S+$ $\gamma)^{-2} S_{x}$ occurring in two-phase flow in porous media", SIAM J. Appl. Math. 42 (1982) 318-332.

[8] D. B. McWhorter, Infiltration affected by flow of air, Hydrology papers 49 (CSU, Fort Collins, Colorado, 1971).

[9] D. B. McWhoner and D. K. Sunada, "Exact integral solutions for two-phase flow", Water Resour. Res. 26 (1990) 399-413.

[10] J.-Y. Parlange, "Theory of water movement in soils. One-dimensional absorption", Soil Sci. 111 (1971) 134-137.

[11] J.-Y. Parlange, "On solving the flow equation in unsaturated soils by optimization: horizontal infiltration", Soil Sci. Soc. Amer. J. Proc. 39 (1975) 415-418.

[12] J.-Y. Parlange and R. D. Braddock, "A note on some similarity solutions of the diffusion equation", J. Appl. Math. Phys. (ZAMP) 31 (1980) 653-656.

[13] J.-Y. Parlange, R. D. Braddock and B. T. Chu, "First integrals of the diffusion equation; An extension of the Fujita solutions", Soil Sci. Soc. Amer. J. 44 (1980) 908-911.

[14] J.-Y. Parlange, R. D. Braddock, G. C. Sander and F. Stagnitti, "Three-dimensional similarity solutions of the nonlinear diffusion equation from optimization and first integrals", J. Austral. Math. Soc. Ser. B 23 (1982) 297-309.

[15] J.-Y. Parlange, R. D. Braddock and G. Voss, "Two-dimensional similarity solutions of the nonlinear diffusion equation from optimization and first integrals", Soil Sci. 131 (1981) 1-8.

[16] C. Rogers, M. P. Stallybrass and D. A. Clement, "On two-phase infiltration under gravity and with boundary infiltration: Application of a Backlünd transformation", Nonlinear Anal., Theory and Appl. 7 (1983) 785-799.

[17] G. C. Sander, J. Norbury and S. W. Weeks, "An exact solution to the nonlinear diffusion-convection equation for two-phase flow", Q. J. Mech. Appl. Math. 46 (1993) 709-727.

[18] G. C. Sander, J.-Y. Parlange and W. L. Hogarth, "Extension of the Fujita solution to air and water movement in soils", Water Resour. Res. 24 (1988) 1187-1191.

[19] G. C. Sander, J.-Y. Parlange, W. L. Hogarth and R. D. Braddock, "Similarity and first integral solutions for air and water diffusion in soils and comparison with optimal results", Soil Sci. 138 (1984) 321-325.

[20] B. E. Sleep and J. F. Sykes, "Compositional simulation of groundwater contamination by organic compounds 2. Model applications”, Water Resour. Res. 29 (1993) 1709-1718.

[21] G. Sposito, "Lie group invariance of the Richards equation", in Dynamics of fluid in hierarchal porous media (ed. J. H. Cushman), (Academic Press, London, 1990) 327-347.

[22] G. Sposito, "Recent advances associated with soil water in the unsaturated zone", Rev. Geophys. Suppl. (1995) 1059-1065.

[23] S. A. Stothoff and G. F. Pinder, "A boundary integral technique for multiple-front simulation of incompressible, immiscible flow in porous media", Water Resour. Res. 28 (1992) 2067-2076.

[24] S. W. Weeks, G. C. Sander, I. G. Lisle and J.-Y. Parlange, "Similarity solutions of radially symmetric two-phase flow", J. Appl. Math. Phys. (ZAMP) 45 (1994) 84I-853. 